\title{
Dynamic behaviour of Josephson-junction qubits: crossover between Rabi oscillations and Landau-Zener transitions
}

\author{
S.N. Shevchenko ${ }^{(1,2)}$, A.S. Kiyko(1), A.N. Omelyanchouk ${ }^{(1)}$, W. Krech ${ }^{(2)}$ \\ (1) B. Verkin Institute for Low Temperature Physics and Engineering, \\ 47 Lenin Ave., 61103, Kharkov, Ukraine. \\ (2) Friedrich Schiller University, Institute for Solid State Physics, \\ Helmholtzweg 5, D-07743 Jena, Germany.
}

(Dated: October 10, 2018)

\begin{abstract}
We study the dynamic behaviour of a quantum two-level system with periodically varying parameters by solving the master equation for the density matrix. Two limiting cases are considered: multiphoton Rabi oscillations and Landau-Zener (LZ) transitions. The approach is applied to the description of the dynamics of superconducting qubits. In particular, the case of the interferometertype charge qubit with periodically varying parameters (gate voltage or magnetic flux) is investigated. The time-averaged energy level populations are calculated as functions of the qubit's control parameters.

PACS numbers: 03.67.Lx, 03.75.Lm, 74.50.+r, 85.25.Am
\end{abstract}

\section{INTRODUCTION}

The quantum two-level system is a model to describe a number of physical objects such as atoms, quantum dots, molecular magnets, etc. The system can be excited from the ground state to the upper state by changing in time its parameters (due to external fields). If the parameters vary adiabatically slow, the excitation mechanism is called Landau-Zener (LZ) transition ${ }^{1}{ }^{2}$; if the amplitude of the field is small and its frequency is comparable to the level distance, Rabi oscillations occur ${ }^{3,4}$. Thus the occupation of the levels in a two-state system can be controlled by several parameters, e.g., by amplitude and frequency of an external field ${ }^{5}$.

Recently, the two-level model was used to describe Josephson-junction systems $\frac{6}{}$, i.e., both charge qubits $\frac{7}{}$ and flux qubits 8 . The subject of the present work is the investigation of the dynamic behaviour of superconducting qubits with periodically swept parameters, which is important from the point of view of state control and readout. We will relate our results to some other articles concerning the resonant excitation of a two-level system ${ }^{9-13}$ which was shown to be relevant for superconducting qubits too 14-17. Particularly, we will describe the dynamic behaviour of the interferometer-type charge qubit $18-21$.

To study theoretically the dynamic behaviour of a Josephson qubit, we make use of the master equation for the density matrix rather than the Schrödinger equation because this allows to take into account both relaxation and non-zero temperature effects. (However, in this paper we will assume the zero-temperature limit, because we are interested in the influence of relaxation processes only on the qubit dynamics.)

The diagonal components of the density matrix define the probabilities of finding the system in the respective states of the basis in which the density matrix is presented. Thus, executing the calculations we ought to deal with a particular basis. Our calculations are mostly carried out in the stationary basis $\{|-\rangle,|+\rangle\}$ of the eigenstates of the Hamiltonian $\widehat{H}^{(0)}$ in the absence of timedependent terms. We do that for two reasons. First, it is convenient to describe Rabi oscillations and multiphoton transitions. Second, in the case of the charge qubit these states $\{|-\rangle,|+\rangle\}$ are eigenstates of the current operator, which is related to the experimentally measurable values 21 . The important point is that we can get the occupation probability of any state provided we know the probabilities for the states in a particular basis. To demonstrate this, we will change over from the stationary basis to the so-called adiabatic basis (consisting of the instantaneous eigenstates of the time-dependent Hamiltonian $\widehat{H}$ ) to describe the LZ effect. We emphasize that the presented results are valid for the description of any two-level system with periodically swept parameters, particularly, of a superconducting qubit. In a Josephsonjunction qubit the gate voltage or the magnetic flux can be modulated periodically.

The paper is organized as following. In Sec. II the basic equations are presented. In Sec. III we study multiphoton processes and LZ transitions in a two-level system with time-dependent parameters. We apply the respective results to the phase-biased charge qubit in Sec. IV.

\section{THE BASIC EQUATIONS}

We start from the Hamiltonian of a two-level system (see e.g. Ref. 6)

$$
\widehat{H}^{(0)}=-\frac{B_{x}^{(0)}}{2} \widehat{\sigma}_{x}-\frac{B_{z}^{(0)}}{2} \widehat{\sigma}_{z}
$$

in the basis of "physical" states $\{|0\rangle,|1\rangle\}$, where $\widehat{\sigma}_{z}|0\rangle=$ $|0\rangle, \widehat{\sigma}_{z}|1\rangle=-|1\rangle, \widehat{\sigma}_{x, y, z}$ are the Pauli matrices. The "physical" states are the eigenstates of the Hamiltonian 
$\widehat{H}^{(0)}$ for $B_{x}^{(0)} / B_{z}^{(0)} \rightarrow 0$. In the case of a charge qubit, these states correspond to a definite number of Cooper pairs on the island. For a flux qubit, they correspond to a definite direction of the current circulating in the ring. The Hamiltonian (1) is diagonalized by means of the matrix

$$
\widehat{S}=\exp \left(i \frac{\lambda}{2} \widehat{\sigma}_{y}\right)=\left[\begin{array}{cc}
\cos \lambda / 2 & \sin \lambda / 2 \\
-\sin \lambda / 2 & \cos \lambda / 2
\end{array}\right]
$$

where

$\sin \lambda=-\frac{B_{x}^{(0)}}{\Delta E}, \quad \cos \lambda=\frac{B_{z}^{(0)}}{\Delta E}, \quad \Delta E=\sqrt{B_{x}^{(0) 2}+B_{z}^{(0) 2}}$.

The eigenstates $|-\rangle$ and $|+\rangle$ of the time-independent Hamiltonian $\widehat{H}^{(0)}$ are connected with the initial basis:

$$
\left[\begin{array}{l}
|-\rangle \\
|+\rangle
\end{array}\right]=\widehat{S}\left[\begin{array}{l}
|0\rangle \\
|1\rangle
\end{array}\right]
$$

Next, we introduce the time-dependent terms into the Hamiltonian (1),

$$
\widehat{H}^{(0)} \rightarrow \widehat{H}=\widehat{H}^{(0)}+\widehat{H}^{(1)}(t) .
$$

We consider two situations,

$(a): \quad B_{x}=B_{x}^{(0)}, \quad B_{z}=B_{z}(t)=B_{z}^{(0)}+B_{z}^{(1)}(t)$,

$(b): \quad B_{z}=B_{z}^{(0)}, \quad B_{x}=B_{x}(t)=B_{x}^{(0)}+B_{x}^{(1)}(t)$,

where the time-independent/dependent terms are marked with $(0) /(1)$ indices. Making use of the transformation $\widehat{H}^{\prime}=\widehat{S}^{-1} \widehat{H} \widehat{S}$, we get the Hamiltonian $\widehat{H}^{\prime}$ in the energy representation $\{|-\rangle,|+\rangle\}$ corresponding to these cases:

$$
\begin{aligned}
& \widehat{H}_{a}^{\prime}=-\frac{\Delta E}{2} \widehat{\tau}_{z}-\frac{B_{z}^{(1)}(t)}{2}\left(\sin \lambda \widehat{\tau}_{x}+\cos \lambda \widehat{\tau}_{z}\right), \\
& \widehat{H}_{b}^{\prime}=-\frac{\Delta E}{2} \widehat{\tau}_{z}-\frac{B_{x}^{(1)}(t)}{2}\left(\cos \lambda \widehat{\tau}_{x}-\sin \lambda \widehat{\tau}_{z}\right) .
\end{aligned}
$$

For convenience, we use different notations for the Pauli matrices, $\widehat{\sigma}_{i}$ and $\widehat{\tau}_{i}$, which operate in the bases $\{|0\rangle,|1\rangle\}$ and $\{|-\rangle,|+\rangle\}$, respectively.

We emphasize that after the substitution

$$
\begin{aligned}
B_{x}^{(1)}(t) & \rightarrow B_{z}^{(1)}(t), \\
B_{z}^{(0)} & \rightarrow-B_{x}^{(0)}, \\
B_{x}^{(0)} & \rightarrow B_{z}^{(0)}
\end{aligned}
$$

the problem (b) coincides with problem (a). This transition from Eq. (5) to Eq. (4) corresponds to a $\pi / 2$ rotation about the $y$-axis.
To unify expressions (4) and (5) to get the equations for numerical calculations, we write down the Hamiltonian $\widehat{H}^{\prime}$ as following:

$$
\widehat{H}^{\prime}=\frac{A}{2} \widehat{\tau}_{x}+\frac{C}{2} \widehat{\tau}_{z}
$$

The quantum dynamics of our two-level system can be characterized within the standard density-matrix $\operatorname{approach}^{22}$. The time-evolution of the total system composed of the two-level system and the reservoir is described by the Liouville equation. After tracing over the reservoir variables, the Liouville equation can be simplified to the so-called master equation for the reduced density matrix $\widehat{\rho}$. It can be written in the form

$$
\widehat{\rho}=\frac{1}{2}\left[\begin{array}{cc}
1+Z & X-i Y \\
X+i Y & 1-Z
\end{array}\right]
$$

which ensures the condition $\operatorname{Tr} \widehat{\rho}=1$. The effect of relaxation processes in the system due to the weak coupling to the reservoir can be phenomenologically described with the dephasing rate $\Gamma_{\phi}$ and the relaxation rate $\Gamma_{\text {relax }}(23)$. Then the master equation takes the form of Bloch equations ${ }^{22}$ :

$$
\begin{aligned}
& \frac{d X}{d t}=-C Y-\Gamma_{\phi} X, \\
& \frac{d Y}{d t}=-A Z+C X-\Gamma_{\phi} Y, \\
& \frac{d Z}{d t}=A Y-\Gamma_{\text {relax }}(Z-Z(0)) .
\end{aligned}
$$

(Throughout the paper we admit $\hbar=1$.) From these equations we get $Z(t)$ which defines the occupation probability of the upper level $|+\rangle, P_{+}(t)=\rho_{22}(t)=\frac{1}{2}(1-$ $Z(t))$. We choose the initial condition to be $X(0)=$ $Y(0)=0, Z(0)=1$, that corresponds to the system in the ground state $|-\rangle$. We have calculated the time evolution of $P_{+}(t)$ (which is essential e.g. for the snap-shot measurements 24$)$ as well as the time-averaged probability $\bar{P}_{+}$(which is essential e.g. for the impedance measurement technique $\left.{ }^{25}\right)$. We note that the asymptotic expression, $\left.P_{+}(t)\right|_{t \rightarrow \infty}$, is periodic in time with the period $T=2 \pi / \omega$ of the time-dependent term of the Hamiltonian $\widehat{H}^{(1)}(t)$ (see Sec. 12 of Ref. 5).

\section{NON-STATIONARY EFFECTS IN A TWO-LEVEL SYSTEM}

\section{A. Rabi oscillations}

Hereafter we will treat the problem (a) (see Eq. (2)), making use of the following notations:

$$
\widehat{H}=\Delta \widehat{\sigma}_{x}+x(t) \widehat{\sigma}_{z}, \quad x(t)=x_{o f f}+x_{0} \sin \omega t
$$


with

$$
\begin{aligned}
B_{x}^{(0)} & =-2 \Delta, \\
B_{z}^{(0)} & =-2 x_{o f f}, \\
B_{z}^{(1)}(t) & =-2 x_{0} \sin \omega t .
\end{aligned}
$$

This reformulation is convenient for comparing our results with the results of other papers $\underline{\underline{9}}-12$. Then the Hamiltonian (44) can be rewritten:

$$
\begin{aligned}
\widehat{H}^{\prime} & =-\frac{\Delta E}{2} \widehat{\tau}_{z}+x_{0} \sin \omega t \cdot \widehat{V} \\
\widehat{V} & =\frac{2 \Delta}{\Delta E} \widehat{\tau}_{x}-\frac{2 x_{o f f}}{\Delta E} \widehat{\tau}_{z} .
\end{aligned}
$$

First consider the situation $x_{o f f}=0$, then the difference between the stationary energy levels is $\Delta E=2 \Delta$. In the case

$$
\Delta \omega \equiv \omega-\Delta E \ll \Delta E \text { and } x_{0} \ll \Delta E,
$$

one can use the so-called rotating wave approximation, and the result is 26

$$
P_{+}(t)=\frac{1}{2} \frac{x_{0}^{2}}{x_{0}^{2}+(\Delta \omega)^{2}}\left(1-\cos \sqrt{x_{0}^{2}+(\Delta \omega)^{2}} t\right)
$$

For the average probability, it follows

$$
\bar{P}_{+}=\frac{1}{2} \frac{x_{0}^{2}}{x_{0}^{2}+(\Delta \omega)^{2}} .
$$

This means that at $\omega=\Delta E$ there is resonance, $\bar{P}_{+}=\frac{1}{2}$, and $P_{+}(t)$ is an oscillating function with the frequency $x_{0}$. This is illustrated in Fig. 17a. The width of the peak at $\omega=\Delta E$ of the $\bar{P}_{+}-\omega$ curve at the half-maximum (i.e., at $P_{+}=1 / 4$ ) is approximately $2 x_{0}$ (see the upper panels of Fig. 2).

Resonant excitations of a two-level system for $x_{0} / \Delta E \ll 1$ may occur not only at $\omega=\Delta E$. In the $K$-th order approximation, $\left(x_{0} / \Delta E\right)^{K}$, resonances exist at $\omega \simeq \Delta E / K^{4}, 27$. For $x_{o f f}=0$, the resonances occur at odd $K$ only. The dependence of $P_{+}$on time in the vicinity of $K$-th resonance is described by a relation similar to Eq. (15) (with substitutions $\Delta \omega \rightarrow \Delta \omega^{(K)} \simeq K \omega-\Delta E$ and $\left.x_{0} \rightarrow x_{0}^{K}\right)$. Hence, the width of the $K$-th resonance is of the order of $x_{0}^{K}$. In the resonance, $\Delta \omega^{(K)}=0$, the energy levels $|-\rangle$ and $|+\rangle$ are equally populated so that $\bar{P}_{+}\left(\omega=\omega^{(K)} \simeq \Delta E / K\right)=1 / 2$, which is the maximum value of $\bar{P}_{+}$, since the population inversion in a two-level system is not possible.

When $x_{\text {off }} \neq 0$, the matrix $\widehat{V}$ has non-zero diagonal elements (see Eq. (13)). The appearance of the non-zero diagonal elements does not influence Rabi resonances at odd $K$, but rather results in the generation of the resonances at even $K$. This is demonstrated in Fig. 2 and discussed in Sec. III.C.
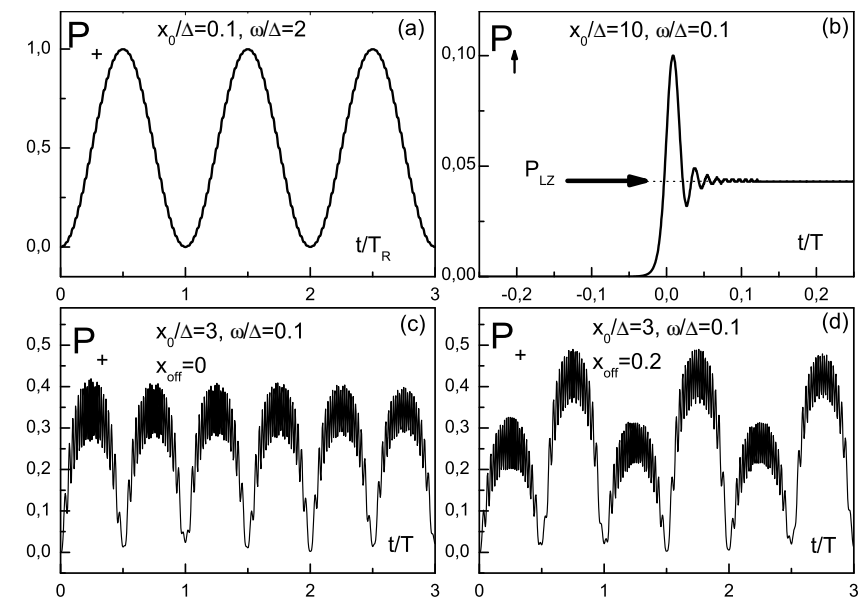

FIG. 1: Time dependence of upper-level occupation probabilities. (a) Rabi oscillations in $P_{+}$with the period $T_{R}=2 \pi / x_{0}$, (b) LZ transition in $P_{\uparrow}$ (see Sec. III.B), (c) and (d) $P_{+}$probability evolution in the case of periodically swept parameters
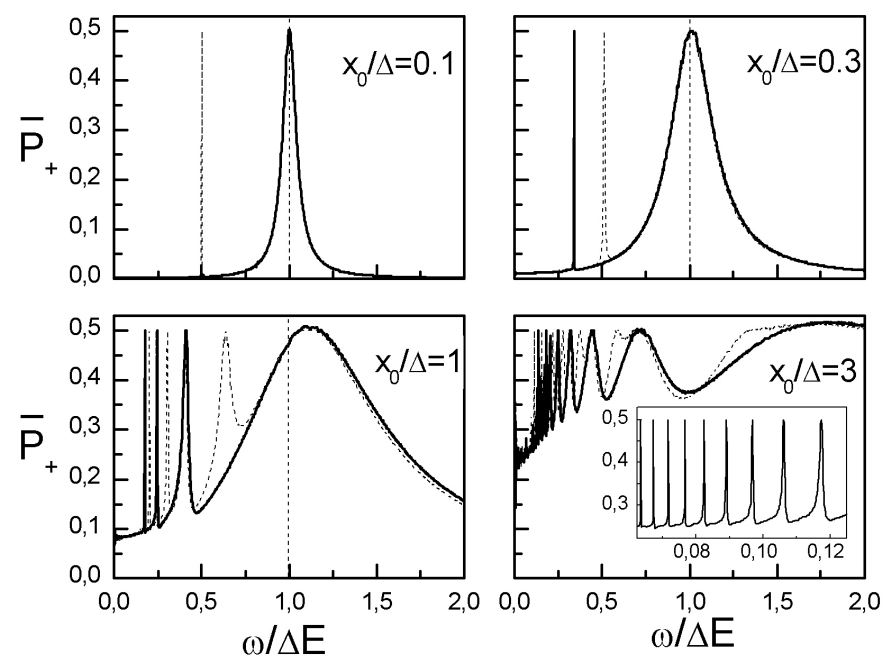

FIG. 2: Dependence of the probability $\bar{P}_{+}$on the frequency $\omega$ for different $x_{0}$ at $\Gamma_{\phi}=\Gamma_{\text {relax }}=0$ and at $x_{o f f}=0$ (solid line) and $x_{o f f}=0.2 \Delta$ (dashed line). (Only the first few resonant peaks are plotted; the others, which are very narrow, are not shown at the graphs.) Inset: enlargement of the lowfrequency region.

\section{B. LZ effect}

The LZ effect manifests in the non-adiabatic transition with the probability

$$
P_{L Z}=\exp \left(-\frac{\pi \Delta^{2}}{\omega x_{0}}\right)
$$

between two adiabatic energy levels during a single-sweep event ${ }^{1}$.

The time-dependent Hamiltonian $\widehat{H}$ is diagonalized in the adiabatic basis, denoted as $\{|\downarrow\rangle,|\uparrow\rangle\}$, by the $\widehat{S}_{1}$ ma- 
trix,

$$
\begin{gathered}
\widehat{S}_{1}=\left[\begin{array}{cc}
\cos \frac{\eta}{2} & \sin \frac{\eta}{2} \\
-\sin \frac{\eta}{2} & \cos \frac{\eta}{2}
\end{array}\right] \\
\sin \eta=-\frac{B_{x}}{\Delta E_{1}}=\frac{2 \Delta}{\Delta E_{1}}, \cos \eta=\frac{B_{z}}{\Delta E_{1}}=-\frac{2 x(t)}{\Delta E_{1}}, \\
\Delta E_{1}(t)=2 \sqrt{\Delta^{2}+x(t)^{2}}
\end{gathered}
$$

The instantaneous eigenvalues of $\widehat{H}$ are $E_{\downarrow, \uparrow}=$ $\pm \Delta E_{1}(t) / 2$.

We now can change over from the stationary basis to the adiabatic one,

$$
\left[\begin{array}{l}
|\downarrow\rangle \\
|\uparrow\rangle
\end{array}\right]=\widehat{S}_{1}\left[\begin{array}{l}
|0\rangle \\
|1\rangle
\end{array}\right]=\widehat{S}_{1} \widehat{S}^{-1}\left[\begin{array}{l}
|-\rangle \\
|+\rangle
\end{array}\right] \equiv \widehat{S}_{2}\left[\begin{array}{l}
|-\rangle \\
|+\rangle
\end{array}\right] .
$$

Assuming $x_{o f f}=0$, we obtain

$$
\widehat{S}_{2}=\frac{1}{\sqrt{2}}\left[\begin{array}{lr}
\cos \frac{\eta}{2}+\sin \frac{\eta}{2} & -\cos \frac{\eta}{2}+\sin \frac{\eta}{2} \\
\cos \frac{\eta}{2}-\sin \frac{\eta}{2} & \cos \frac{\eta}{2}+\sin \frac{\eta}{2}
\end{array}\right] .
$$

Thus, provided we calculate the density matrix in the stationary basis $\widehat{\rho}$, we find it in the adiabatic basis $\widehat{\rho}_{\text {adiab }}$,

$$
\widehat{\rho}_{a d i a b}=\widehat{S}_{2}^{-1} \widehat{\rho}_{2} .
$$

The initial condition for $\widehat{\rho}$ can be obtained from the initial condition for $\widehat{\rho}_{a d i a b}$ by inverting the relation (18).

Let us now consider as an illustrative example the onesweep process,

$$
\begin{aligned}
t & \in\left(-\frac{T}{4}, \frac{T}{4}\right), T=\frac{2 \pi}{\omega}, \\
x(t) & =x_{0} \sin \omega t, x(t) \in\left(-x_{0}, x_{0}\right), \\
x_{0} & \gg \Delta \gg \omega,
\end{aligned}
$$

which corresponds to the LZ model28. We choose the initial condition to be

$$
\widehat{\rho}_{\text {adiab }}\left(-\frac{T}{4}\right)=\left[\begin{array}{ll}
1 & 0 \\
0 & 0
\end{array}\right],
$$

which means that the system at $t=-T / 4$ is in the lower adiabatic state $|\downarrow\rangle$. We look for the occupation probability $P_{\uparrow}=\rho_{\text {adiab }}^{22}(t)$ of the upper adiabatic level $|\uparrow\rangle$, which equals to the LZ probability at the end of the sweep ${ }^{28}$, $P_{\uparrow}(T / 4)=P_{L Z}$. Thus, for the functions $X, Y, Z$, introduced in Sec. II, we get the initial condition

$$
\begin{aligned}
& X\left(-\frac{T}{4}\right)=\frac{x_{0}}{\sqrt{\Delta^{2}+x_{0}^{2}}}, Y\left(-\frac{T}{4}\right)=0, \\
& Z\left(-\frac{T}{4}\right)=\frac{\Delta}{\sqrt{\Delta^{2}+x_{0}^{2}}}
\end{aligned}
$$

and find

$$
\begin{aligned}
P_{\uparrow}(t)= & \frac{1}{2}-\frac{\Delta}{2 \sqrt{\Delta^{2}+x(t)^{2}}} Z(t)+ \\
& +\frac{x(t)}{2 \sqrt{\Delta^{2}+x(t)^{2}}} X(t) .
\end{aligned}
$$

This is illustrated in Fig. 10 which is equivalent to Fig. $3 \mathrm{~d}$ in Ref. 10.

In the general case, $x_{o f f} \neq 0$, we calculate $\widehat{S}_{2}$ and then $\widehat{\rho}_{\text {adiab }}$ according to Eq. (18). Then the probability $P_{\uparrow}$ is given by

$$
\begin{aligned}
P_{\uparrow}(t)= & \frac{1}{2}-\frac{\left(\Delta^{2}+x(t) x_{o f f}\right)}{2 \sqrt{\Delta^{2}+x(t)^{2}} \sqrt{\Delta^{2}+x_{o f f}^{2}}} Z(t)+ \\
& +\frac{\left(\Delta x(t)-\Delta x_{o f f}\right)}{2 \sqrt{\Delta^{2}+x(t)^{2}} \sqrt{\Delta^{2}+x_{o f f}^{2}}} X(t) .
\end{aligned}
$$

This should be supplemented with the respective initial condition.

\section{Crossover from multiphoton Rabi resonances to LZ-interferometry: numerical results}

Now making use of the numerical solution of Eqs. 8 10) for the Hamiltonian (13), we study the dependence of the time-averaged probability $\bar{P}_{+}$on frequency $\omega$ and amplitude $x_{0}$. For small amplitudes, $x_{0} \ll \Delta E$, there are resonant peaks in the $\bar{P}_{+}-\omega$ dependence at $\omega \simeq \Delta E / K$, as described in Sec. III.A and illustrated in Fig. 2 With increasing amplitude $x_{0}$, the resonances shift to higher frequencies. For $x_{o f f}=0$, the resonances appear at "odd" frequencies $(K=1,3,5, \ldots)$ only, as it was studied in Ref. 9. For $x_{o f f} \neq 0$ there are also resonances at "even" frequencies $(K=2,4, \ldots)$, which is demonstrated in Fig. 2] We note that Fig. 2] is plotted for the ideal case of the absence of decoherence and relaxation, $\Gamma_{\phi}=\Gamma_{\text {relax }}=0$, when the resonant value is $\bar{P}_{+}=1 / 2$. The effect of finite dephasing, $\Gamma_{\phi} \neq 0$, and relaxation, $\Gamma_{\text {relax }} \neq 0$, is to decrease the resonant values of $\bar{P}_{+}$and to widen the peaks for $\Gamma_{\phi}>\Gamma_{\text {relax }}$. Thus from the comparison of the theoretically calculated resonant peaks with the experimentally observed ones, the dephasing $\Gamma_{\phi}$ and the relaxation rates $\Gamma_{\text {relax }}$ can be obtained $^{16}$.

When the system is driven slowly, $\omega \ll \Delta$, and with large amplitude, $x_{0} \gg \Delta$, the LZ excitation mechanism is relevant for the description of the system dynamics. In the previous subsection we considered the LZ transition for a single-sweep event. Now we study the periodical driving of the system: Interferences between multiple LZ transitions happen, which leads to resonant excitations $13,12,2$. We will compare these resonances with the multiphoton Rabi ones.

First, we note that the resonance positions depend on the amplitude; we demonstrate that for $\omega \ll \Delta<x_{0}$ in the inset of Fig. [2] But it is more illustrative to study the resonance properties via the dependence of $\bar{P}_{+}$ on the ratio $x_{0} / \omega$ (or, more precisely, on the phase the state vector picks up per half-period $\left.{ }^{12} \phi=4 x_{0} / \omega\right)$. It is reasonable to carry out the calculation for a fixed value of the product $x_{0} \omega$, which in its turn defines the LZprobability (see Eq. (17)). This allows us not only to 

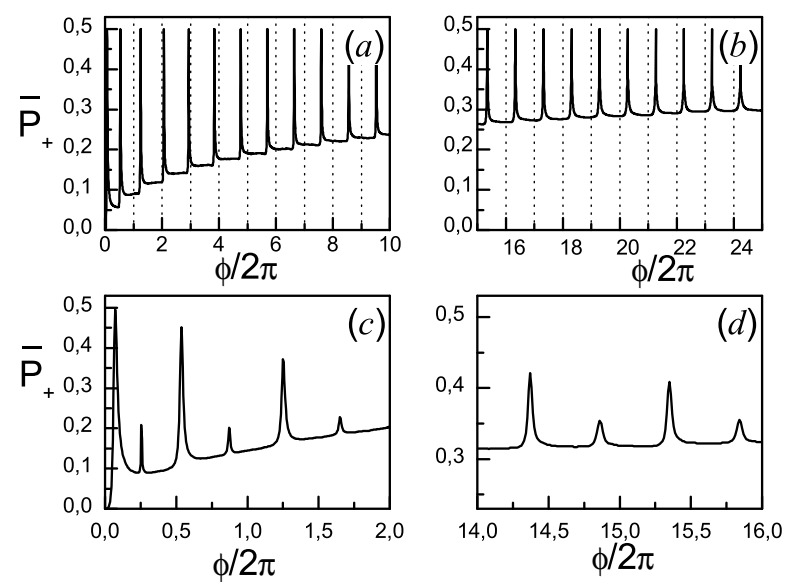

FIG. 3: Dependence of the probability $\bar{P}_{+}$on $\phi=4 x_{0} / \omega$ at $x_{0} \omega / \Delta^{2}=0.45$ (which corresponds to $P_{L Z}=10^{-3}$ ); $\Gamma_{\phi}=$ $\Gamma_{\text {relax }}=0$ and $x_{o f f}=0$ for graphs (a) and (b); $\Gamma_{\phi} / \Delta=$ $\Gamma_{\text {relax }} / \Delta=10^{-3}$ and $x_{o f f} / \Delta=0.05$ for graphs (c) and (d).

compare our results with the results of Ref. 12 , where the periodicity of the resonances in $\phi$ was predicted, but also to demonstrate the transition from the multiphoton Rabi resonances to the ones induced due to the interference of LZ excitations by means of the $\bar{P}_{+}-\phi$ dependence.

In Fig. 3(a,b) we plotted the dependence of $\bar{P}_{+}$on $\phi$ for $x_{o f f}=0$ and $\Gamma_{\phi}=\Gamma_{\text {relax }}=0$. The first few peaks, in the region $x_{0} \lesssim \Delta$, corresponding to the multiphoton Rabi resonances are situated at $\phi \sim K^{2}$ (that follows from the resonance relation $\omega=\Delta E / K)$. With increasing $\phi$ (which is proportional to $x_{0}$ ) we observe the overall rising of the curve in accordance with the conclusions of Ref. 9. At $\phi \gg 1$, i.e., at $\omega \ll \Delta \ll x_{0}$, the resonance position is $2 \pi$-periodic in agreement with Ref. 12. The non-zero offset $x_{o f f} \neq 0$ results in the appearance of additional resonances between the basic ones 12 , which is demonstrated in Fig. 3( $\mathrm{c}, \mathrm{d})$. Such a feature is similar to the multiphoton Rabi resonances. Nonzero decoherence decreases and widens the peaks we have also demonstrated in Fig. 31(c,d).

In Fig. 4 we present the dependence of $\bar{P}_{\uparrow}$ on $\phi$ for the calculations carried out in the adiabatic basis (cf. Sec. III.B). In Fig. 4(a,b) we plotted such a dependence for different values of $\phi$ at $x_{o f f}=0$ and $\Gamma_{\phi}=\Gamma_{\text {relax }}=0$. For $\phi \gg 1$ (see Fig. 40) the resonances are $2 \pi$-periodic, and the peaks are situated at the integer values of $\phi / 2 \pi$, as predicted in Ref. 12. We also plotted the dependence of $\bar{P}_{\uparrow}$ on $\phi$ for the non-zero offset $x_{o f f} \neq 0$ (see Fig. 目(c,d)), namely for $\phi_{o f f}=\pi / 2$. For this value the additional peaks become as high as the basic ones.
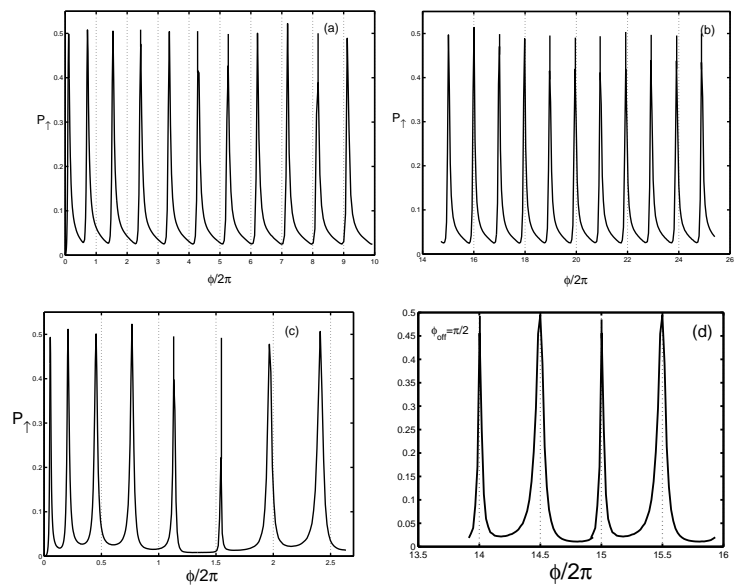

FIG. 4: The dependence of the probability $\bar{P}_{\uparrow}$ on the phase $\phi$.

\section{MULTIPHOTON EXCITATIONS IN THE INTERFEROMETER-TYPE CHARGE QUBIT}

\section{A. Interferometer-type charge qubit}

In this section, we consider the quantum dynamics of the interferometer-type charge qubit with periodically varying control parameters $19-21$. This qubit consists of two Josephson junctions closed by a superconducting ring. The charge en of the island between the junctions is controlled by the gate voltage $V_{g}$ via the capacitance $C_{g}$. The junctions are characterized by Josephson energies $E_{J 1}, E_{J 2}$ and phase differences $\varphi_{1}, \varphi_{2}$. The relevant energy values are the island's Coulomb energy, $E_{C}=e^{2} / 2 C_{t o t}$, where $C_{t o t}$ is the total capacitance of the island, and the effective Josephson energy

$$
\varepsilon_{J}=\left(E_{J 1}^{2}+E_{J 2}^{2}+2 E_{J 1} E_{J 2} \cos \delta\right)^{1 / 2} .
$$

An important feature of the qubit is that its Josephson energy is controlled by the external magnetic flux $\Phi_{e}$ piercing the ring. In this paper, the ring inductance $L$ is assumed to be small. Then the total phase difference, $\delta=\varphi_{1}+\varphi_{2}$, approximately equals to $\delta_{e}=2 \pi \Phi_{e} / \Phi_{0}$, and thus $\varepsilon_{J}=\varepsilon_{J}(\delta) \simeq \varepsilon_{J}\left(\delta_{e}\right)$.

Within the two-level model with the basis of "charge states" $|0\rangle$ and $|1\rangle$ corresponding to the number of Cooper pairs on the island, the Hamiltonian of the interferometer-type charge qubit can be written as ${ }^{6}$

$$
\widehat{H}=-\frac{1}{2} \varepsilon_{J} \widehat{\sigma}_{x}-\frac{1}{2} E_{c h} \widehat{\sigma}_{z},
$$

where $E_{c h}=4 E_{C}\left(1-n_{g}\right)$ and $n_{g}=C_{g} V_{g} / e$. Here the domination of the Coulomb energy of a Cooper pair $4 E_{C}$ over the coupling energy $\varepsilon_{J}$ is assumed, $4 E_{C} / \varepsilon_{J}>1$. The eigenstates, $\{|-\rangle,|+\rangle\}$, of this Hamiltonian are discriminated by the direction of the supercurrent in the ring 21 . 
The qubit is considered to be coupled not only to the gate but also to the tank circuit that enables both phase control and readout. Thus there are two possibilities to make the Hamiltonian of the two-level system (19) timedependent. First, the gate voltage $V_{g}$ can be driven,

$$
n_{g}=n_{g}^{(0)}+n_{g}^{(1)} \sin \omega t,
$$

and, second, the DC and $\mathrm{AC}$ components of the current in the tank circuit can induce a periodically varying magnetic flux,

$$
\delta=\delta_{D C}+\delta_{A C} \sin \omega t .
$$

Here we restrict our consideration to the case of the sinusoidal time dependence of the parameters.

Further we study the time-averaged occupation probability of the excited state $\bar{P}_{+}$. We note that because the two states $|-\rangle$ and $|+\rangle$ belong to oppositely circulating currents in the ring, they correspond to different signs of the qubit's Josephson inductance. The latter can be probed by the impedance measuring technique ${ }^{25}$, which makes it possible to observe the resonant behaviour of $\bar{P}_{+}$studied in the following subsection.

\section{B. Resonant excitation of the interferometer-type charge qubit}

The case of the excitation of the interferometer-type charge qubit via the gate (Eq. (201) can easily be related to the one considered in Sec. III; see the Hamiltonians (11) and (19) with the diagonal time-dependent parameters defined by Eq. (20). Then the ratio $x_{0} / \Delta$ is given by

$$
\frac{x_{0}}{\Delta}=\frac{4 E_{C}}{\varepsilon_{J}} n_{g}^{(1)}
$$

Thus, both mechanisms considered in Sec. III can be realized: multiphoton excitations $\left(x_{0} / \Delta \ll 1\right)$ and LZ interferometry $\left(x_{0} / \Delta \gg 1\right)$.

Let us now consider the second possibility of the excitation of the qubit by the time-dependent magnetic flux. The Hamiltonian of the interferometer-type charge qubit (19) with the periodically varying phase $\delta$ (Eq. (21) $)$ is related to the Hamiltonian of a two-level system considered in Sec. II (Eq. (5) ) by the following relations:

$$
\begin{aligned}
B_{x}(t) & \equiv \varepsilon_{J}, \\
B_{x}^{(0)} & =\left.B_{x}(0) \equiv B_{x}\right|_{\delta_{A C}=0}, \\
B_{x}^{(1)}(t) & =B_{x}(t)-B_{x}(0), \\
B_{z}^{(0)} & =E_{c h} .
\end{aligned}
$$

In two limiting cases, the expressions can be simplified to result the time-dependent term in the form

$$
B_{x}^{(1)}(t) \propto \sin \omega t
$$

Namely, for

$$
\delta-\pi \gg \frac{\left|E_{J 1}-E_{J 2}\right|}{\sqrt{E_{J 1} E_{J 2}}}, \delta_{A C} \lesssim \delta_{D C}-\pi \ll \pi
$$

we have

$$
\begin{aligned}
B_{x}^{(0)} & \simeq \sqrt{E_{J 1} E_{J 2}}\left(\delta_{D C}-\pi\right), \\
B_{x}^{(1)}(t) & \simeq \sqrt{E_{J 1} E_{J 2}} \delta_{A C} \sin \omega t,
\end{aligned}
$$

and for

$$
\delta-\pi \ll \frac{\left|E_{J 1}-E_{J 2}\right|}{\sqrt{E_{J 1} E_{J 2}}}, \delta_{A C} \ll \delta_{D C}-\pi \ll \pi
$$

it follows

$$
\begin{aligned}
B_{x}^{(0)} & \simeq\left|E_{J 1}-E_{J 2}\right|, \\
B_{x}^{(1)}(t) & \simeq \frac{E_{J 1} E_{J 2}}{\left|E_{J 1}-E_{J 2}\right|}\left(\delta_{D C}-\pi\right) \delta_{A C} \sin \omega t .
\end{aligned}
$$

When the relation (22) takes place, the present problem (of the charge qubit with time-dependent magnetic control) can be related to the problem considered above in Sec. III by the expressions (6). Then we can estimate the ratio $x_{0} / \Delta E$, which characterizes the mechanism of the excitation of the qubit. E.g., at $x_{0} / \Delta E \lesssim 1$ we expect multiphoton resonances in the qubit's response to the external alternating magnetic flux. We consider this case below in detail.

We note that the width of the resonant peaks is defined by the non-diagonal components of the Hamiltonian (13), i.e. by the product $x_{0} \Delta$. Then looking at the Eqs. (25) and (28), we conclude that the width of the resonances is defined by the product $\delta_{A C}\left(1-n_{g}\right)$.

Finally, we illustrate the multiphoton resonant excitations of the interferometer-type charge qubit. Making use of the numerical solution of the master equation (Sec. II), we find the time-averaged probability $\bar{P}_{+}$plotted in Fig. 5. The position of the multiphoton resonant peaks is defined by the relation $\omega=\Delta E / K$, where $\Delta E=\Delta E\left(\delta_{D C}\right)$ is supposed to be fixed. Alternatively, when the $\delta_{D C}$ component of the phase is changed and the frequency $\omega$ is fixed, a similar graph can be plotted with resonances at $\delta_{D C}=\delta_{D C}^{(K)}$ defined by the relation $\Delta E\left(\delta_{D C}^{(K)}\right)=K \omega$.

\section{CONCLUSIONS}

We have studied the dynamic behaviour of a quantum two-level system subjected to periodical sweeping of its parameters. The energy levels population was calculated by solving the master equation for the density matrix with relaxation terms. Studying the population of the excited state in both stationary and adiabatic bases, we analyzed some features of the multiphoton Rabi and LZ effects. Particularly, we have shown certain similarities of the multiphoton resonances at $x_{0} \ll \omega$ with the resonances at $x_{0} \gg \Delta \gg \omega$ due to the interference between 


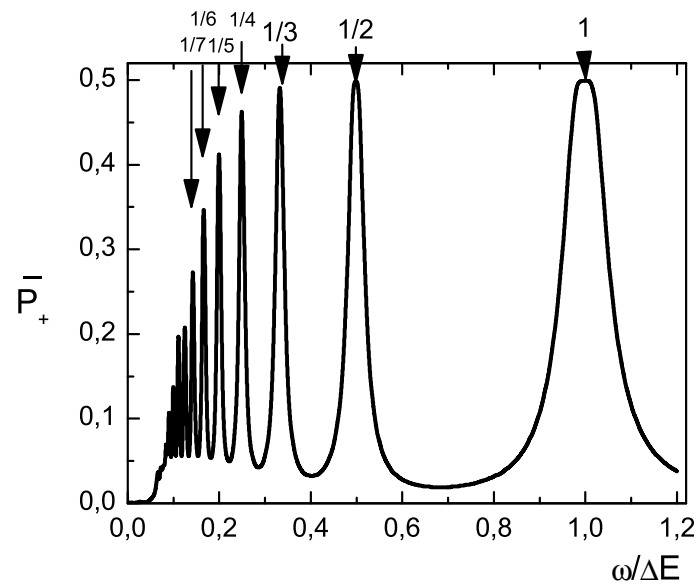

FIG. 5: Dependence of the probability $\bar{P}_{+}$on the frequency $\omega$ for the phase-biased charge qubit at $n_{g}=0.95, E_{J 1} / E_{C}=$ $12.4, E_{J 2} / E_{C}=11, \Gamma_{\phi} / E_{C}=5 \cdot 10^{-4}, \Gamma_{\text {relax }} / E_{C}=10^{-4}$, $\delta_{A C}=0.2 \pi, \delta_{D C}=\pi+0.2 \pi$. multiple LZ transitions. Based on the solution of the master equation for the density matrix, we described in detail the dynamic behaviour of the interferometer-type charge qubit subjected to periodically changing gate voltage or magnetic flux.
1 L.D. Landau, Phys. Z. Sowjetunion 2, 46 (1932); C. Zener, Proc. R. Soc. London, Ser. A 137, 696 (1932).

${ }^{2}$ V.A. Benderskii, E.V. Vetoshkin, and E.I. Kats, JETP 97, 232 (2003).

3 I.I. Rabi, Phys. Rev. 51, 652 (1937).

4 N.B. Delone and V.P. Krainov, Atoms in Strong Light Fields, Springer Ser. Chem. Phys., Vol. 28, Springer, Berlin-Heidelberg (1985) (also in Russian: Atom v sil'nom svetovom pole, Atomizdat, Moscow (1978)).

${ }^{5}$ M. Grifoni and P. Hänggi, Phys. Rep. 304, 229 (1998).

6 Yu. Makhlin, G. Schön and A. Shnirman, Rev. Mod. Phys. 73, 357 (2001).

7 Y. Nakamura, Yu.A. Pashkin, and J.S. Tsai, Nature 398, 786 (1999).

8 J.E. Mooij et al., Science 285, 1036 (1999).

9 V.P. Krainov and V.P. Yakovlev, Sov. Zh. Exp. Theor. Fiz. 78, 2204 (1980).

10 N.V. Vitanov, Phys. Rev. A 59, 988 (1999).

11 D.A. Garanin and R. Schilling, Phys. Rev. B 66, 174438 (2002).

12 A.V. Shytov, D.A. Ivanov, and M.V. Feigel'man, cond-mat/0110490 v2.

13 Y. Teranishi and H. Nakamura, Phys. Rev. Lett. 81, 2032 (1998).

14 Y. Nakamura, Yu.A. Pashkin, and J.S. Tsai, Phys. Rev. Lett. 87, 246601 (2001).

15 J.Q. You and F. Nori, Phys. Rev. B 68, 064509 (2003).

16 S. Saito et al., Phys. Rev. Lett. 93, 037001 (2004).
17 D. Born et al., Phys. Rev. B 70, 180501(R) (2004).

18 G. Falci et al., Nature 407, 355 (2000).

19 A.B. Zorin, Physica C 368, 284 (2002).

20 J.R. Friedman and D.V. Averin, Phys. Rev. Lett. 88, 050403 (2002).

${ }^{21}$ W. Krech et al., Phys. Lett. A 303, 352 (2002).

22 K. Blum, Density Matrix Theory and Applications, Plenum Press, New York-London (1981).

23 The phenomenological description of the relaxation processes in terms of the rates was shown ${ }^{12}$ to be equivalent to the microscopic description which intrinsically includes the reservoir. Such a phenomenological approach was successfully applied to the description of the quantum dynamics of phase qubits 16 .

24 A. Aassime et al., Phys. Rev. Lett. 86, 3376 (2001).

25 E. Il'ichev et al., Low Temp. Phys. 30 , 620 (2004).

26 S. Flügge, Practical Quantum Mechanics II, Springer, Berlin-Heidelberg-New York (1971), task 180.

27 C. Cohen-Tannoudji, J. Dupont-Roc, and G. Grynberg, Atom-Photon Interactions, Wiley, New York (1992).

28 In the vicinity of the transition point $x=0$ (where the excitation happens), $x(t)$ is a linear function of $t, x(t) \simeq x_{0} \omega t$, which is exactly the LZ model. Deviations of the function $x(t)$ from the linear dependence far from the transition point do not matter ${ }^{11}$. This explains the quantitative coincidence of the results presented in this section with the results obtained with $x(t)$ being a linear function. 